\title{
Inteligencia emocional y adaptabilidad social en mandos gerenciales
}

\section{Emotional intelligence and social adaptability in management controls}

Cinthia Marlene Hernández-López

Universidad de Guadalajara
Georgina Lozano-Razo

Universidad Autónoma de Zacatecas

\section{Resumen}

El objetivo de la presente investigación es conocer si existe relación estadísticamente significativa entre Inteligencia Emocional (IE) y Adaptabilidad Social (AS) en trabajadores con un puesto gerencial, debido a que se ha demostrado que la IE se relaciona con el reconocimiento y regulación de las expresiones emocionales de los otros (Fernández \& Extremera, 2009), favorece el desarrollo de habilidades prácticas y de manejo del estrés, un mayor bienestar percibido y la mejora de la calidad de los servicios ofrecidos a los usuarios (Extremera, Durán, \& Rey, 2005). El presente estudio es de tipo correlacional, para el cual se tomó una muestra no probabilística intencional de 50 gerentes de sucursales de algunas de las empresas de Fresnillo, Zacatecas. Dicha muestra se evaluó mediante la aplicación simultánea de la versión Española del Trait Meta Mood Scale TMMS-24 (Fernández, Extremera, \& Ramos, 2004) y el Cuestionario de Adaptabilidad Social MOSS elaborado por Rudolf Moss y Berenice Moss en 1979. Los datos se analizaron con el paquete estadístico SPSS, versión 15.0, así se obtuvo el coeficiente de correlación paramétrico de Pearson. Los resultados arrojados muestran que existe una relación positiva, baja y significativa $(r=.512, p=0.05)$, entre la habilidad de supervisión y la atención emocional.

Palabras clave: inteligencia emocional, adaptabilidad social, psicología organizacional, reclutamiento y selección, recursos humanos.

Nota del autor

Cinthia Marlene Hernández-López, Centro Universitario de Ciencias de la Salud, Universidad de Guadalajara; Georgina Lozano-Razo, Universidad Autónoma de Zacatecas.

La correspondencia en relación con este artículo debe dirigirse a Cinthia Marlene Hernández-López, Centro Universitario de Ciencias de la Salud, Universidad de Guadalajara, Ónix \#538, Villa Esmeralda, C.P. 45654, Tlajomulco de Zúñiga, Jalisco, México.

Dirección electrónica: psicmarhe@outlook.com 


\begin{abstract}
The aim of this research is to determine whether there is a statistically significant relationship between IE and AS in workers with a management position because, it has been shown that IE is related to the recognition and regulation of emotional expressions of others (Fernández \& Extremera, 2009), promotes the development of practical skills and stress management, greater perceived wellbeing and improving the quality of services offered to users (Extremera, Duran, \& King, 2005). The present study is correlational, for which an intentional non-probabilistic sample was selected from 50 company managers in Fresnillo, Zacatecas. The sample was evaluated by the simultaneous application of Spanish version of the Trait Meta Mood Scale TMMS-24 (Fernández, Extremera, \& Ramos, 2004) and the Social Adaptability Questionnaire developed by Rudolf Moss MOSS and Berenice Moss in 1979. Data were analyzed with SPSS version 15.0 to obtain the parametric correlation coefficient of Pearson. Results show that there is a positive, low and significant relationship $(r=.512, p=0.05)$ between the supervisory ability and emotional care.
\end{abstract}

Keywords: emotional intelligence, social adaptability, organizational psychology, recruitment and selection, human resources.

El concepto de Inteligencia Emocional (IE) es introducido por Salovey y Mayer (1997) quienes la definieron como la habilidad de percibir, asimilar y comprender los sentimientos propios y los de los demás, así como distinguir entre ellos con el fin solucionar problemas y adaptarse de manera eficaz al medio.

A raíz del surgimiento del concepto de IE otros autores han optado por elaborar su propio modelo, de ahí la variabilidad de éstos. Trujillo y Rivas (2005) nos señalan en su documento Orígenes, evolución y modelos de inteligencia emocional dos tipos de modelos, a saber, el modelo mixto, que se relaciona con los rasgos de personalidad, y el modelo de habilidades. Dentro de los llamados modelos mixtos, de acuerdo a los autores mencionados encontramos, El modelo de Bar-On, que toma en cuenta las habilidades intrapersonales, habilidades interpersonales, adaptabilidad, manejo de estrés y estado anímico general; de acuerdo con dicho modelo, la IE es un conjunto de capacidades emocionales, personales e interpersonales que influyen en la capacidad del individuo para hacer frente a las demandas del medio ambiente (Bar On, 2006). El modelo de Goleman (1995), que a su vez incluye cinco componentes: autoconocimiento, autorregulación, empatía, motivación y habilidades sociales. Y finalmente, el modelo de El EQ-Map de Oriolo y Cooper (1998), que evalúa al hombre en su integralidad y engloba cinco aptitudes: entorno habitual, conciencia emocional, competencias, valores y actitudes.

En cuanto a los modelos de habilidades encontramos el Modelo de Salovey y Mayer (1997), que nos habla de cuatro habilidades esenciales de la IE, como la percepción 
emocional, asimilación emocional, comprensión emocional y la regulación emocional.

Como hemos visto desde la aparición del concepto de IE han surgido una variedad de modelos y se han suscitado con ellos numerosas investigaciones, sobre todo en el área educativa, donde se le ha correlacionado con diversas variables; entre las más controvertidas se encuentran, su semejanza con rasgos de personalidad y capacidades cognitivas (Pena $\&$ Repetto, 2008). Sin embargo, a pesar de que abundan las investigaciones en torno a la IE (Fernández \& Extremera, 2006; Padrón \& Sánchez, 2010; Bernarás, Garaigordobil, \& De las Cuevas 2011), y debido a que ha demostrado relaciones significativas con distintas variables como rendimiento académico (Ferragut \& Fierro, 2012), autoeficacia (Perandones \& Castejón, 2007), personalidad, adaptación social, bienestar (Fierro, 2006) liderazgo (Zarate \& Matviuk, 2012) y felicidad (Fernández \& Extremera, 2009), por mencionar algunas, es necesario retomarla en el área laboral, sobre todo en la dirección de recursos humanos. Independientemente de que sea considerado un constructo cognitivo o de personalidad existe relativamente pocas investigaciones realizadas en América Latina sobre temas de carácter organizacional y laboral (Zarate \& Matviuk, 2012). Tal y como mencionan Trujillo y Rivas (2005), "La mayor parte de la literatura y las investigaciones publicadas sobre IE están en el campo de la educación... el campo de las organizaciones ha sido muy pobremente explorado" (p. 22).

Dentro de los estudios realizados en el área laboral encontramos el de Veloso, Cuadra, Antezana, Avendaño y Fuentes (2013), quienes plantean en su investigación realizada con una muestra de trabajadores de educación especial, que la IE correlaciona positivamente con satisfacción vital; se señala que la calidad de vida laboral se ve favorecida por el fomento de habilidades emocionales, ya que dichas habilidades "pueden permitir el ajuste del pensamiento en las tareas cognitivas y a su vez, generar un adecuado desempeño en la ejecución de actividades asociadas a la práctica profesional" (Alesa \& García, 2010, p. 230). Según estos resultados y en lo expuesto por otros autores (Extremera et al., 2005), la IE favorece el desarrollo de habilidades prácticas y de manejo del estrés, un mayor bienestar percibido y la mejora de la calidad de los servicios ofrecidos a los usuarios.

De los resultados obtenidos en otras investigaciones (Vera, Cancino, Ravanal, Carrasco, Contreras, \& Arteaga, 2007) se ha descubierto que existe una relación entre IE, burnout y factores de riesgos laborales. Asimismo, Ortiz y Beltrán (2011) proporcionan evidencias de correlaciones de los componentes de la IE, del modelo de habilidades de Salovey y Mayer, con factores de desgaste laboral. En este contexto, Extremera y Fernández (2004) identifican problemas que son provocados por la 
deficiencia de IE, entre los que figuran el déficit en los niveles de bienestar, ajuste psicológico y la disminución en la cantidad y calidad de las relaciones interpersonales.

Aunado a lo anterior, Sanjuán y Ferrer (2008), en su investigación realizada con una muestra de practicantes médicos, concluyen que los sujetos con mayor déficit en el control y manejo de las emociones, perciben como más negativo el ambiente de las prácticas clínicas y la guía prestada por otros para la realización de las mismas. Sin embargo, esto nos lleva a plantearnos la siguiente pregunta: ¿qué variable en relación con la IE, puede ayudarnos a esbozar mejores propuestas para el área de capacitación y selección de puestos, y por ende, tener mayor impacto en la planeación, organización, dirección y control de la empresa? Respecto a este punto, estudios como el de Fierro (2006) sugieren una investigación a profundidad de la relación entre la IE con la AS, ya que ambas variables podrían tener relación con el buen manejo de los recursos humanos, puesto que comprenden aspectos como la habilidad para detectar, evaluar y establecer relaciones interpersonales, resolver problemas derivados de los mismos, capacidad para la supervisión y guía del personal, habilidad de detección y regulación de estados emocionales propios, así como del personal a cargo.

\section{IE como componente importante del perfil gerencial}

Para esta investigación se eligió el modelo de habilidades de Salovey y Mayer (1997), adaptado por Fernández y Extremera (2009), ya que es el modelo que ha sido más ampliamente aceptado y utilizado en esta área (Trujillo \& Rivas 2005). Dichos autores plantean que la IE está basada en el uso adaptativo de las emociones, de manera que el individuo puede solucionar problemas y adaptarse de forma eficaz al medio que le rodea (Fernández \& Ruiz, 2008). Esta manera de ver la IE subraya la habilidad de adaptación a un ambiente en constante cambio (Sternberg \& Kaufman, 1998, como se citó en Fernández \& Extremera, 2009), adaptación necesaria para el establecimiento de las relaciones socio-laborales.

El modelo de Salovey y Mayer (1997) nos habla de cuatro habilidades esenciales de la IE, éstas son expuestas por Fernández y Extremera (2009): la percepción emocional, habilidad que nos permite identificar las emociones, sensaciones fisiológicas y cognitivas propias y discriminar acertadamente las emociones expresadas por los demás; la asimilación emocional, que es la habilidad para reconocer cómo las emociones influyen en la manera de procesar la información y en la toma de decisiones; la comprensión emocional, que comprende diversas destrezas entre ellas rotular las emociones, identificar aquellos estímulos 
que provocan estados emocionales específicos, conocer las consecuencias de nuestras acciones con base en los estados emocionales que éstas pueden generar, identificar emociones secundarias, habilidad para descifrar emociones complejas, y pericia para reconocer emociones contradictorias, así como los cambios de uno a otro estado emocional; la última habilidad que comprende este modelo es la regulación emocional, que de acuerdo a Fernández y Extremera (2009), es la habilidad más compleja de la IE y abarca factores como la capacidad de aprovechar la información emocional para regular las emociones propias y de otros, al intensificar las emociones positivas y mermar las negativas.

La adaptación de Fernández y Extremera (2006), considera solo tres variables clave de la IE: atención emocional, que es capacidad de sentir y expresar sentimientos adecuadamente y reconocer e interpretar el significado de los mismos; la claridad emocional, que involucra la comprensión sobre cómo se crean las emociones complejas a partir de las básicas, cómo las emociones son influidas por los acontecimientos, y cómo las reacciones emocionales se suscitan en determinados entornos sociales (Alasa \& García, 2010); y la reparación emocional, que implica la capacidad de regular estados emocionales propios y de otros correctamente.

El concepto de IE como se ha mencionado, ha sido ampliamente utilizado en la literatura y propuesto como una inteligencia esencial en el desarrollo académico, psicológico, social y laboral del sujeto. En cuanto al área laboral, de acuerdo a Goleman (1999), 73\% las aptitudes consideradas fundamentales para el desarrollo exitoso de un trabajador que se desenvuelve en una organización se refiere a aptitudes emocionales. Además, la IE se podría relacionar más estrechamente con los profesionales que la requieren en mayor grado, entre ellos, como sugiere Enebral (2001), los directivos; por el contrario, los informáticos, los técnicos de laboratorio o los contables no precisan tanto de ésta.

Aunque la interacción interpersonal influya en la práctica de la IE, el procesamiento de la información emocional "puede permitir el ajuste del pensamiento en las tareas cognitivas $\mathrm{y}$, a su vez, generar un adecuado desempeño en la ejecución de actividades asociadas a la práctica profesional" (Alasa \& García, 2010, p. 230). Por tanto, ¿la IE podría relacionarse directamente con el modo de interacción gerente-subordinado, y en la manera en que el gerente se desempeña en sus actividades? Bajo esta lógica, es necesario revisar las aptitudes del perfil gerencial, que podrían considerarse como aptitudes basadas en las habilidades emocionales propuestas por Fernández y Extremera (2009) del modelo de Salovey y Mayer (1997).

\section{La adaptabilidad (AS)}

En cuanto al concepto de adaptación este alude a "la necesidad que tiene el individuo de lograr un ajuste entre sus propias características personales, incluyendo sus necesidades, y las 
demandas del medio donde interactúa" (Bosque \& Aragón. 2008). Por su parte, Pisquiy (2012 como se citó en Subuyuj, 2013), define la A ${ }^{1}$ S como:

El nivel, más o menos óptimo, de acomodación comportamental al modo de vida existente y mayoritariamente aceptado en el grupo al que se pertenece, así como la disposición del individuo para participar activamente en dicho grupo y de esa forma adaptarse socialmente al mismo. (p. 34).

De este concepto podemos encontrar componentes, retomados y considerados como unidades de adaptabilidad que permiten a las personas ajustarse de manera efectiva, éstos factores son la flexibilidad cognoscitiva, flexibilidad emocional y disposición al cambio (Melo, 2013, como se citó en Subuyuj, 2013).

Por lo tanto, la AS en los mandos directivos y gerenciales involucraría aspectos como la motivación, misma que permita ejercer esfuerzo a favor de las metas organizacionales, en compaginación con las necesidades individuales de los subordinados, la asimilación de las normas y políticas de la organización, y el cambio conductual generado a partir de ellas y de las pautas implícitas de las relaciones laborales interpersonales; el ajuste cognitivo y emocional, derivado de estas relaciones interpersonales y

1 De acuerdo con la Real Academia Española (2015), el concepto de adaptabilidad alude a la capacidad de adaptarse, mientras que el concepto adaptación es la acción o el efecto, por lo tanto, a lo largo del documento nos referiremos a la adaptabilidad como una cualidad del sujeto. procedente del procesamiento de información social en conexión con el nivel de la IE del sujeto (Robbins, 1999). Es aquí donde la AS y la IE tienen en común el procesamiento de la información social con ayuda de la información emocional (proveniente del mismo sujeto y de los otros), que influye en la emisión de una respuesta cognitiva, emocional y/o conductual.

Gran parte del desarrollo de la empresa es generado por los mandos gerenciales, ya que en ellos recae la dirección de la empresa material y la dirección de los recursos humanos, por lo cual la AS generada a partir de las normas de la empresa y las pautas implícitas de las relaciones interpersonales no es suficiente, puesto que el nivel de la AS debe mostrar niveles altos de las habilidades necesarias para cubrir el perfil. Entre éstas últimas, la habilidad para la supervisión, la toma de decisiones en las relaciones humanas, la capacidad de evaluación de problemas interpersonales, la habilidad para establecer relaciones interpersonales, el sentido común y tacto, necesarias para el cumplimiento eficaz de las funciones directivas del personal a cargo.

\section{Desarrollo de competencias a nivel directivo y gerencial}

Crissien (2005) define el término gerencia como el arte de maximizar el desarrollo del recurso humano, y de los otros recursos con que se cuenta. Este autor hace una aclaración entre los componentes de la gerencia y expone que los recursos se administran y la gente se 
gestiona o lidera. Explica que el perfil de un gerente-líder debe ser integral, es decir, implica el desarrollo de una serie de competencias, entre ellas las conceptuales: consisten en ser un buen investigador de la especie humana, tener un pensamiento estratégico y anticipado; las competencias técnicas: incluyen el dominio de los procesos administrativos, que según Centina, Ortega y Aguilar (2010), se refieren a las habilidades de mercado, que comprenden las capacidades necesarias para competir y encargarse de las relaciones externas, así como mantener el control y estabilidad general de la empresa; y las humanísticas: consisten en la capacidad de empoderar, motivar y capacitar a los subordinados, así como observar y desarrollar sus potencialidades (Crissien, 2005). De igual manera, Hernández Silvestri, Añez y Gamboa (2008) proponen una serie de capacidades que deben formar parte del perfil gerencial: entendimiento del entorno, situaciones y elementos relevantes para su uso en la elaboración de respuestas adecuadas a la realidad organizacional; flexibilidad de pensamiento, que permita interpretar la realidad de diferentes enfoques y visualizar diferentes alternativas; construcción de una visión compartida que funcione de motivación; aprendizaje en equipo y potencialización del aprendizaje del personal mediante la confianza e intercambio de experiencias.

En este contexto, las competencias gerenciales no solo deberían incluir las competencias conceptuales, técnicas y humanísticas, ya que dentro de este perfil se hacen necesarias las competencias emocionales, que junto con las humanísticas y sociales se complementen para el manejo eficiente del personal y mejora en los procesos de pensamiento, así como para la toma de decisiones (procesos donde participan las competencias técnicas y conceptuales).

Como se ha visto, gran parte del desarrollo de la empresa depende de la dirección de los mandos gerenciales, ya que en ellos recae el manejo de los recursos humanos, por lo tanto, los programas de capacitación gerencial deben ir orientados al perfeccionamiento de estas dimensiones, considerando el desarrollo de la IE como parte primordial del desarrollo gerencial.

\section{Métodos de selección como una problemática en la contratación de puestos directivos y ge- renciales}

Los métodos de selección de personal se definen principalmente por las políticas de administración de recursos humanos en la empresa (Aguilar, Ambrocio, \& Vargas, 2011). Sin embargo, a lo largo de los años las empresas han dado prioridad al factor económico como fuente de competitividad y no al capital intelectual como un factor determinante en el éxito de la misma (Calderón, 2003). Debido a lo mencionado, las compañías han descuidado sus procesos de selección de personal, y provocado así que dichos procesos conlleven paradójicamente, un aumento de costos, debido a que los puestos son ocupados por personas que no cumplen el perfil. 
Según Chiavenato (2000), el proceso de selección es el medio para que la organización cumpla sus metas, por lo cual debe incluir: análisis de los requerimientos del puesto (con base en un análisis de puesto correctamente realizado), descripción del perfil a cubrir, elección del método de reclutamiento, realización de entrevista y aplicación de técnicas de selección adecuadas al perfil, elaboración de informe final y entrevista. No obstante, los métodos de selección de personal no corresponden a estas características; de acuerdo a un estudio hecho en Oaxaca, más de 50\% de la selección del personal se realiza por recomendación, 30\% no realiza exámenes de selección, y en $25 \%$ de los casos no se cuenta con un análisis de puesto con el cual comparar los resultados (Aguilar et al., 2011). Es importante entonces cuestionar, si los métodos de selección de personal y el proceso mismo cumplen con el objetivo o sólo son trámites para el llenado de vacantes. En este mismo estudio, dan un listado de formas distintas de selección de personal, entre las cuales se encuentran: la entrevista, verificación de antecedentes, pruebas psicométricas y seguimiento de redes sociales como Facebook y Twitter.

Si nos basamos en los porcentajes antes mencionados, observamos que sólo $30 \%$ de esos solicitantes realiza exámenes psicométricos, sin embargo, desconocemos qué tipo de pruebas realizan y qué porcentaje de los resultados se compara con un perfil detallado. De igual manera, no sabemos si estos perfiles consideran variables como la IE y AS. Por ello, si se pre- tende el aumento de la competitividad de la empresa es necesario que se ponga más énfasis en el método de selección del personal, sobre todo en la selección de mandos gerenciales, puesto que son ellos los encargados de la dirección de la empresa y de la coordinación del personal.

A continuación se ejemplificará cómo es que las personas pueden realizar cierto manejo en la impresión que dan en las entrevistas de selección de personal, en base en el video nombrado "el candidato" y considerando los componentes de la IE presentados por Fernández y Extremera (2009). En este video la empresa cervecera Heineken utiliza un modelo innovador en la selección de su capital humano, al basarse en el employer brainding ${ }^{3}$ y muestra cómo se expone al solicitante a diferentes contingencias para evaluar su reacción y determinar si es adecuado o no para el puesto.

Tomaré como ejemplo el segundo test expuesto en el video (min 1:12), aplicado a los postulantes, llamado "asistencia médica". En esta prueba el entrevistador aparenta sentirse mal, el solicitante del puesto utiliza la primera variable de la IE, la percepción emocional, para identificar las emociones, sensaciones fisiológi2 Consultar video en https://www.youtube.com/ watch? $\mathrm{v}=\mathrm{KkAlRZ8F} 4 \mathrm{LI}$

3 El employer branding se define como "una estrategia a largo plazo dirigida a gestionar el conocimiento y las percepciones de los empleados -actuales y potenciales- acerca de una empresa en particular" (Sullivan, 2004 como se citó en Blasco, Fernández, \& Rodríguez, 2014, p. 34) y de acuerdo a Belichón (2006), incluye características como reputación de la empresa, posibilidad de desarrollo profesional, buenas retribuciones, una buena cultura corporativa y un buen capital intelectual. 
cas propias y de la persona que supone sentirse mal (es importante mencionar que las variables de la IE sólo son utilizadas en medida del nivel ya desarrollado por el sujeto, por consiguiente, esta misma percepción emocional puede o no ser eficaz en cada uno de los postulantes, lo cual impide el uso adecuado de los ulteriores componentes de la IE).

Posteriormente, el entrevistador trata de levantarse y cae, en esta parte se pone en práctica la asimilación emocional, en lo referente a la habilidad para reconocer cómo las emociones influyen en la manera de procesar la información y tomar decisiones (componente imprescindible para la emisión de una respuesta conductual y cognitiva en lo referente a las acciones y la toma de decisiones respecto a la situación). En el video se observan distintas conductas en cada uno de los candidatos, siendo la más ilustrativa la de la mujer que elige ir a la puerta en búsqueda de ayuda, pero antes de salir cambia de opinión y decide socorrer al entrevistado. Es aquí donde la comprensión emocional le permite a cada uno de los postulantes conocer las consecuencias de su acción con base en los estados emocionales que presentaron, para consecuentemente identificar emociones secundarias al suceso y aprovechar la información emocional percibida, asimilada y comprendida con el fin de regular sus emociones y las del entrevistador, al tiempo que disminuye las negativas y potencializa las positivas, para así entrar nuevamente en el ciclo.

Con todo lo anterior, el objetivo de este estudio es conocer si existe relación estadísticamente significativa entre IE y AS en trabajadores con un puesto gerencial.

\section{Las hipótesis estadísticas}

Hipótesis nula: no existe relación estadísticamente significativa entre la IE y la AS en trabajadores con un puesto gerencial.

Hipótesis alternativa: sí existe relación estadísticamente significativa entre la IE y la AS en trabajadores con un puesto gerencial.

\section{Método}

\section{Participantes}

El presente estudio es de tipo correlacional, para su realización se tomó una muestra no probabilística intencional, constituida por $50 \mathrm{su}-$ jetos trabajadores, todos ellos pertenecientes a mandos gerenciales de algunas de las sucursales de empresas ubicadas en Fresnillo, Zacatecas; del total 31 fueron de sexo masculino y 19 de sexo femenino, cuyas edades oscilaban entre 22 y 52 años.

\section{Instrumentos}

Para el estudio de las variables se evaluó dicha muestra, mediante la aplicación de la versión española del Trait Meta Mood Scale TMMS24 (Fernández, Extremera, \& Ramos, 2004), que evalúa el metaconocimiento de los estados emocionales en tres secciones importantes: atención emocional, claridad de sentimientos $y$ reparación emocional (con un alfa de Cronbach 
para percepción de 0.90; comprensión, 0.90; y regulación, 0.86).

También se aplicó el Cuestionario de Adaptabilidad Social MOSS, elaborado por Rudolf Moss y Berenice Moss en 1979, con una adaptación latina en 1989, el cual determina el grado en que una persona se adapta a distintas situaciones sociales, comprende los siguientes factores: habilidad en supervisión, capacidad de decisión en las relaciones humanas, capacidad de evaluación de problemas interpersonales, habilidad para establecer relaciones interpersonales, sentido común y tacto en las relaciones interpersonales. Dichos factores de juicio social se califican con los siguientes niveles: deficiente, pobre, medio, bueno $\mathrm{y}$ superior.

\section{Procedimiento}

La aplicación de ambos test se realizó de manera simultánea e individual, se acudió a cada una de las sucursales de las empresas, donde se eligió un espacio sin distractores. La aplicación de ambos test tuvo una duración de entre $40 \mathrm{y}$ 55 minutos. En dicha aplicación se registraron datos como la escolaridad, el nombre de la empresa, el género, tipo de carrera cursada (en caso de haber alguna), el puesto y el número de personal a cargo.

Dentro de las empresas que colaboraron con la investigación se cuentan sucursales bancarias y crediticias como Banamex, Santander, Caja Popular Mexicana, Banco Azteca, HSBC
México, Scotiabank, Banorte, Bbva Bancomer, Concrédito, Unimex, Financiera Independencia, Banco del Bajío; tiendas departamentales, entre ellas Vision, Coppel, Bodega Aurrera, Waldos Dólar Mart, Soriana, Elecktra del milenio; restaurantes como Garden's restaurant, Restaurante el viejo mercado; así como tiendas que venden productos textiles y de vestir, entre ellas Modatelas, Santory, Atletas Fresnillo, E1 porvenir, Parisina, Grupo Eleczion, Diseño y moda para zoy, Melody, Milano Melody; empresas que brindan servicios como Hotel del Mineral, La Plata Habitaciones, Multiempeños, Hotel Casa Blanca, Tv Zac, Cablecom, Cinemex; zapaterías, entre ellas Zapatera 3 Hermanos, Hermanos Batta, Coppel Canadá, Zapatería Pague Menos, Zapatería El Gran Remate; así como sucursales de empresas con venta de productos específicos como Aucaza, Grupo Centro Aluminios y Motos del Mineral.

Una de las dificultades que se presentaron fue la disponibilidad de tiempo de los gerentes, debido a sus múltiples ocupaciones. Nueve sucursales, entre ellas bancarias y de servicios, se negaron debido al tiempo de aplicación de los test. Asimismo, tres empresas, dos de venta de productos específicos y una de servicios, se negaron a participar por políticas empresariales.

\section{Análisis de datos}

Se realizaron análisis de frecuencia para las variables descriptivas de escolaridad, nombre de la empresa, género, tipo de carrera cursada, el puesto y el número de personal a cargo. Tam- 
bién se realizaron comparaciones de porcentajes de subescalas correspondientes a los dos instrumentos utilizados por género.

Los datos obtenidos de las puntuaciones de los factores de las pruebas, se trataron mediante el paquete estadístico SPSS, versión 15.0, de donde se obtuvo el coeficiente de correlación paramétrico de Pearson con una significancia estadística de $p<0.05$. El método de tal coeficiente se utiliza para determinar si existe asociación lineal entre dos variables cuantitativas continuas, ya sea positiva o negativa. Con este método lo que se obtiene es un estimado del coeficiente de correlación poblacional (Camacho, 2008).

\section{Resultados}

A continuación se reportan los resultados, en un principio los obtenidos respecto a los datos generales de la muestra. En este sentido, 31 sujetos pertenecen al género masculino, 19 pertenecen al género femenino; del total de la muestra, 15 personas son gerentes o directores de sucursales bancarias y financieras, en tanto 13 son gerentes de tiendas departamentales, 9 corresponden a empresas que brindan servicios, entre ellas, hoteles, restaurantes, televisoras, hostales y cine, y los 13 restantes son gerentes a cargo de sucursales de venta de productos específicos, entre ellos zapaterías, autos, motos, ropa $\mathrm{y}$ telas.

Asimismo, 26 sujetos de la muestra poseen una escolaridad a nivel licenciatura, con predo- minio de la carrera en Administración de Empresas, 9 se encuentra en nivel preparatoria, 4 tienen carrera técnica, 8 nivel secundaria y solo 3 sujetos cursaban un posgrado. En cuanto a la cantidad de personal que dirigen, encontramos que 47 sujetos tienen a su cargo a menos de 100 trabajadores y solo 3 supervisan a entre 100 y 250 empleados.

Respecto a los resultados del cuestionario MOSS, $42 \%$ de la muestra total calificó con nivel medio inferior, $56 \%$, con nivel medio; y $2 \%$, con medio superior. No se obtuvo ningún porcentaje en las categorías de inferior y superior.

Los resultados generales del MOSS arrojaron que en habilidad de supervisión, 70\% se encontró de rango medio inferior a inferior y $2 \%$ no calificó ni siquiera como inferior. En capacidad de evaluación de problemas interpersonales se encontró que $50 \%$ se ubicó en los niveles de medio inferior a inferior y 4\% no calificó. En capacidad de evaluación de problemas interpersonales, $36 \%$ se situó en rango inferior, mientras que en habilidad para establecer relaciones interpersonales se encontró que $40 \%$ se colocó en el rango medio inferior a inferior, y $4 \%$ no calificó. Finalmente, en sentido común y tacto en las relaciones interpersonales encontramos que $40 \%$ se posicionó en nivel medio inferior a inferior y $6 \%$ no calificó.

En los resultados de los factores del MOSS por género, se encontró que en el género femenino respecto a la habilidad de supervisión, 57. 9\% se clasificó en el nivel medio inferior, mientras que $26.3 \%$, en el medio superior. En 
cuanto a la capacidad de decisión en las relaciones interpersonales, $52.6 \%$ calificó en el nivel medio y $21.1 \%$, en inferior. Respecto a la capacidad de evaluación de problemas interpersonales, $42.1 \%$ se consideró como medio, solo $5.3 \%$, de nivel inferior. Respecto a la habili- dad para establecer relaciones interpersonales, $47.4 \%$ se concentró en el nivel medio inferior. En sentido común y tacto en las relaciones interpersonales solo 5.3\% calificó en nivel superior, concentrándose $36.8 \%$ en el nivel inferior (ver tabla 1).

Tabla 1

Factores de AS en hombres y mujeres con mandos gerenciales y directivos

\begin{tabular}{lllllll}
\hline \multirow{2}{*}{ Género } & Rango & HS & CDRI & CEPI & HERI & SCTRI \\
\hline \multirow{3}{*}{ Femenino } & Superior & $0 \%$ & $0 \%$ & $0 \%$ & $0 \%$ & $5.3 \%$ \\
& Medio Superior & $26.3 \%$ & $0 \%$ & $15.8 \%$ & $26.3 \%$ & $5.3 \%$ \\
& Medio & $5.3 \%$ & $52.6 \%$ & $36.8 \%$ & $47.4 \%$ & $31.6 \%$ \\
& Medio inferior & $57.9 \%$ & $26.3 \%$ & $5.3 \%$ & $0 \%$ & $21.1 \%$ \\
& Inferior & $10.5 \%$ & $21.1 \%$ & $0 \%$ & $0 \%$ & $36.8 \%$ \\
& & & & & & \\
Masculino & Superior & $0 \%$ & $0 \%$ & $12.9 \%$ & $6.5 \%$ & $3.2 \%$ \\
& Medio Superior & $9.7 \%$ & $16.1 \%$ & $22.6 \%$ & $6.1 \%$ & $6.5 \%$ \\
& Medio & $16.1 \%$ & $25.8 \%$ & $19.4 \%$ & $35.5 \%$ & $19.4 \%$ \\
& Medio inferior & $64.5 \%$ & $35.5 \%$ & $12.9 \%$ & $19.4 \%$ & $38.7 \%$ \\
& Inferior & $6.5 \%$ & $16.1 \%$ & $0 \%$ & $6.1 \%$ & $22.6 \%$ \\
& No califica & $3.7 \%$ & $6.6 \%$ & $0 \%$ & $6.5 \%$ & $9.7 \%$ \\
\hline
\end{tabular}

Nota: Habilidad de Supervisión (HS), Capacidad de Decisión en las Relaciones Interpersonales (CDRI), Capacidad de Evaluación de los Problemas Interpersonales (CEPI), Habilidad para Establecer Relaciones Interpersonales (HERI) y Sentido Común y Tacto en las Relaciones Interpersonales (SCTRI).

En lo que concierne al género masculino, se encontró que en los factores de habilidad de supervisión, $64.5 \%$ calificó en el nivel medio inferior, en tanto que $9.7 \%$ no lo hizo así ni siquiera en el nivel inferior. En cuanto a la capacidad de decisión en las relaciones interpersonales, $35.5 \%$ calificó en el nivel medio inferior y $6.5 \%$ no resultó calificable. Respecto a la capacidad de evaluación de problemas interpersonales, $32.3 \%$ se ubicó en nivel medio, solo $12 \%$ en nivel superior. En cuanto a la habilidad para establecer relaciones interpersonales, $35.5 \%$ se concentró en el nivel medio. En sentido común y tacto en las relaciones interpersonales, solo $5.2 \%$ se ubicó en nivel superior, $64.4 \%$ se concentró en el nivel medio inferior. 
En lo referente a los resultados de IE, se obtuvo que $66 \%$ de la muestra total debe mejorar su atención, ya que presta poca atención (ver tabla 2).

Como se muestra en la tabla anterior, 26\% de la muestra total debería mejorar su claridad y $14 \%$ poseía excelente claridad. Respecto a la reparación, $8 \%$ debería mejorar su reparación y $44 \%$ tenía una excelente reparación.
En cuanto a los resultados por género, se obtuvo que el factor de atención emocional, 78\% de las mujeres prestó poca atención a sus emociones. En el factor claridad emocional, 15.8\% debería mejorar su claridad emocional y $10.5 \%$ presentó una excelente claridad. En reparación emocional, se encontró que 5.3\% debería mejorar su reparación y $63.2 \%$ mostró una excelente reparación (ver tabla 3 ).

Tabla 2

Calificación de las áreas de IE de la muestra total

\begin{tabular}{llll}
\hline \multirow{2}{*}{ Áreas de la IE } & \multicolumn{2}{c}{ Calificación } \\
\hline \multirow{2}{*}{$\mathrm{AE}$} & Presta poca atención & Adecuada & Presta demasiada atención \\
& $66 \%$ & $34 \%$ & $0 \%$ \\
$\mathrm{CE}$ & Debe mejorar & Adecuada & Excelente \\
& $26 \%$ & $60 \%$ & $14 \%$ \\
$\mathrm{RE}$ & Debe mejorar & Adecuada & Excelente \\
\hline
\end{tabular}

Nota: Atención Emocional (AE), Claridad Emocional (CE), Regulación Emocional (RE).

Tabla 3

Calificación de las áreas de IE por género

\begin{tabular}{ccccc}
\hline \multirow{2}{*}{ Género } & \multirow{2}{*}{ Áreas de la IE } & \multicolumn{2}{c}{ Calificación } \\
& & Presta poca atención & Adecuada & Presta demasiada atención \\
\hline Femenino & AE & $78.9 \%$ & $21.1 \%$ & $0 \%$ \\
& & Debe mejorar & Adecuada & Excelente \\
& CE & $15.8 \%$ & $73.7 \%$ & $10.5 \%$ \\
& & Debe mejorar & Adecuada & Excelente \\
& RE & $5.3 \%$ & $31.6 \%$ & $63.2 \%$ \\
& AE & Presta poca atención & Adecuada & Presta demasiada atención \\
& & $58.1 \%$ & $41.9 \%$ & $0 \%$ \\
Masculino & CE & $32.3 \%$ & Adecuada & Excelente \\
& & Debe mejorar & $51.6 \%$ & $16.1 \%$ \\
& RE & $9.7 \%$ & Adecuada & Excelente \\
\hline
\end{tabular}

Nota: Atención Emocional (AE), Claridad Emocional (CE), Regulación Emocional (RE). 
Como se aprecia en la tabla 3 para el género masculino en los factores de la IE, se encontró que $58.1 \%$ prestó poca atención a sus emociones y $41.9 \%$ mostró una adecuada atención. Por su parte, en el factor claridad emocional, 32.3\% debería mejorar su claridad emocional y $16.1 \%$ presentó una excelente claridad. En reparación emocional, se encontró que 9.7\% debería mejo- rar su reparación y $32.3 \%$ poseía una excelente reparación.

Los resultados que arrojaron las correlaciones de los instrumentos de IE y AS, solo mostraron una relación del .512 entre la habilidad de supervisión del cuestionario MOSS y la atención emocional del test TMSS-24, con una significancia estadística de $p<0.05$ (tabla 4).

Tabla 4

Puntajes de correlación de variables

\begin{tabular}{|c|c|c|c|}
\hline \multirow[b]{2}{*}{ Variables } & \multicolumn{3}{|c|}{ Variables } \\
\hline & AE de TMMS-24 & CE de TMMS-24 & Años escolaridad \\
\hline HS del MOSS & $.512^{*}$ & & \\
\hline Años Escolaridad & $.314^{*}$ & $.335^{*}$ & \\
\hline SCT del MOSS & & & $.286^{*}$ \\
\hline CEPI de MOSS & & & $.279^{*}$ \\
\hline
\end{tabular}

Nota: Significancia de $p<0.05$. Atención Emocional (AE), Claridad Emocional (CE), Capacidad de Evaluacion de Problemas Interpersonales (CEPI), Sentido Comun y Tacto (SCT).

Respecto a las correlaciones entre personal a cargo y las variables de la IE y AS, no se encontró relación significativa, sin embargo, se determinó una débil, aunque significativa correlación de .335 entre años de escolaridad y claridad emocional (tabla 4). De igual manera existe una débil correlación de .286 entre escolaridad en años y sentido común y tacto en las relaciones interpersonales. Asimismo, se determinó una correlación de .279 entre escolaridad en años y capacidad de evaluación de problemas interpersonales (ver tabla 4).

\section{Discusión}

El objetivo principal del presente estudio fue dilucidar si existe relación estadísticamente significativa entre IE y AS en trabajadores con un puesto gerencial, con el fin de contribuir al desarrollo e implementación de medidas para el aumento de la calidad de interacción interpersonal dentro de la empresa, el mejoramiento de los procesos de capacitación y selección de puestos directivos y gerenciales. Los resultados obtenidos arrojaron que únicamente el factor de atención emocional de la IE y el factor de 
habilidad de supervisión de la AS expresan una correlación de .512 con una significancia de $p<$ 0.05. Por consiguiente, la hipótesis alternativa se acepta sí existe relación baja y estadísticamente significativa entre la IE y la AS en trabajadores con un puesto gerencial, empero, ésta se limita exclusivamente a las características de la muestra.

Entonces, podemos preguntar, ¿cuál es la explicación de que los factores de habilidad de supervisión y atención emocional hayan mostrado relación estadísticamente significativa? Esto podría deberse a que la habilidad de supervisión es el proceso mediante el cual el gerente conduce las energías, potencialidades y actividades del grupo para alcanzar los objetivos organizacionales. De tal forma, tal habilidad se clasifica dentro de las competencias humanísticas, que incluyen la capacidad de empoderar, motivar y capacitar a los subordinados, así como observar y desarrollar sus potencialidades (Crissien, 2005). Por su parte, la atención emocional, se presenta en niveles poco adecuados y podría repercutir en la capacidad de reconocer, sentir y expresar sentimientos de manera afín con el entorno laboral, además de influir en la forma de dar órdenes al personal, supervisar el desempeño laboral y el cumplimiento de tareas laborales. Asimismo, debe orientar las potencialidades de su personal hacia áreas específicas sin inmiscuir emociones propias en el proceso, dificultar la asignación de tareas de acuerdo con las potencialidades de los trabajadores e influir en los procesos cognitivos relacionados con la toma de decisiones. En consecuencia, las habilidades del supervisor incluyen elaborar un plan de trabajo en correspondencia con los objetivos que se persiguen en la acción por medio de buenas relaciones humanas, para que así se pueda crear un clima organizacional armónico que contribuya al crecimiento del personal (Rodríguez, 2008)

En otros estudios (Subuyuj, 2013), al igual que en el presente, se ha encontrado una diferencia por género en el factor habilidad de supervisión, donde las mujeres han obtenido puntajes más altos que los hombres. Dicha diferencia se podría explicar porque a lo largo de la historia las mujeres han llevado a cabo tareas como la educación de los hijos, la atención de enfermos y de la pareja y la orientación de los miembros de la familia (Aguilar, Valdez, González-López, \& González, 2013). Es de tal manera como las mujeres desarrollan variables comportamentales, sentimentales y de pensamiento que se deben a la influencia de la cultura (Aguilar et al., 2013) y que se relacionan con la habilidad de supervisión.

De acuerdo a Cuadra y Veloso (2009), el grado de supervisión es una variable moderadora de liderazgo general, satisfacción laboral y clima organizacional. La supervisión, por consiguiente, es estrecha (el líder frecuentemente tiene contacto directo con sus empleados) e influye de manera más significativa en el aumento de estas variables y la supervisión lejana (el líder de manera constante tiene contacto indi- 
recto con sus empleados), la que influye en la merma de las mismas. Es por tanto preocupante, que los resultados del factor habilidad de supervisión hayan arrojado en gran porcentaje de la muestra rangos por debajo de la media. Por lo anteriormente expuesto, la habilidad de supervisión es una prioridad en la capacitación gerencial de estas empresas si se desea el aumento de las variables mencionadas.

Por tanto, ¿cómo podría explicarse la falta de relación entre los factores restantes de la AS con los de la IE? En cuanto a la regulación emocional (variable considerada como la más compleja en el modelo de Fernández y Extremera), contrariamente a lo que se podría esperar y de acuerdo con la definición de regulación emocional de Fernandez y Extremera (2009):

...la capacidad para estar abierto a los sentimientos, tanto positivos como negativos, y reflexionar sobre los mismos para descartar o aprovechar la información que los acompaña en función de su utilidad... el manejo de nuestro mundo intrapersonal y también el interpersonal, esto es, la capacidad para regular las emociones de los demás, poniendo en práctica diversas estrategias de regulación emocional que modifican tanto nuestros sentimientos como los de los demás. (p. 93).

Los resultados de los factores restantes de la AS como capacidad de evaluación de problemas interpersonales, sentido común y tacto en las relaciones interpersonales y habilidad para establecer relaciones interpersonales -todas ellas involucradas en lo concerniente al juicio social-, no muestran relaciones significativas con el constructor de regulación emocional anteriormente citado, lo cual podría implicar que la IE solo juega un papel modulador en el inicio del procesamiento de la información (atención emocional) en las relaciones socio-laborales. Por lo tanto, se recomienda explorar tal aspecto en posteriores investigaciones.

La falta de relación entre los factores restantes de la AS con los de la IE podría deberse también a diferentes variables, entre ellas las metodológicas y las intrínsecas a los resultados. En las metodológicas encontramos que la escala de MOSS es un instrumento que no se encuentra contextualizado con el tiempo y cultura de la población mexicana. Tal característica denota la importancia de la creación de escalas de AS, que vayan de acuerdo con los nuevos parámetros establecidos por las recientes investigaciones en el ámbito de las relaciones socio-laborales. Asimismo, que correspondan a las características socioculturales de la población mexicana. De igual manera, la escala de TMMS-24 mide la metacognición de los estados emocionales y no directamente la IE como tal, por lo cual de acuerdo a Fernández y Extremera (2009), dichas medidas autoinformadas poseen sesgos que responden a la deseabilidad social, el solapamiento con medidas de personalidad y su varianza con otras variables auto-informadas como la depresión y la ansiedad, entre otras. 
Respecto al concepto de metacognición, como es ampliamente conocido en el ámbito de la psicología del aprendizaje, se refiere a la "cognición sobre la cognición”, es decir, la autoconciencia del propio procesamiento cognitivo. Dicha autoconciencia no sólo aporta información propia, sino también sobre las personas, la tarea y las estrategias de almacenaje de información metacognitiva. Debido a que es un proceso de autoconocimiento, es importante entonces cuestionar qué tan viable es la medición de la IE mediante una escala autoinformada, ya que según Brown (1985, como se citó en Crespo, 2000), "el conocimiento metacognitivo es falible en el sentido de que puede estar equivocado. Un sujeto puede creer diversas cosas respecto a su cognición, sin embargo, si éstas se contrastan con un determinado criterio podrían resultar de por sí erróneas" (párr., 74).

En cuanto a las variables intrínsecas de la investigación y aludiendo al nivel de estudios de la muestra, por la importancia que se sabe tiene dentro del desarrollo psico-social, se pone de relieve el hecho de que gran número de sujetos no poseelos estudios suficientes para desempeñar las funciones gerenciales. Lo anterior presenta una dificultad; de acuerdo a Hernández et al. (2008), la gran parte de conocimientos necesarios para la actividad gerencial son derivados del curso de una carrera universitaria. Sin mencionar el hecho de que algunos sujetos estudiaron carreras universitarias que no se relacionan con la administración empresarial, podría decirse, entonces, que presentarían dificultades en las competencias técnicas y conceptuales.

Al referirnos a la falta de habilidades en correspondencia con la baja escolaridad, podríamos conjeturar de acuerdo con los resultados, que existe una relación entre la capacidad de evaluación de problemas interpersonales con la escolaridad en años. Tal capacidad implica la comprensión de los problemas, el establecimiento de una buena comunicación, la escucha activa y el reconocimiento de los intereses, así como las destrezas interpersonales e intelectuales para estudiar los problemas y encontrar soluciones, habilidades que pudieran desarrollarse en el proceso educativo. De igual manera existe relación en el sentido común y tacto en las relaciones interpersonales con los subalternos y la escolaridad, puesto que conlleva la capacidad de tomar en cuenta las habilidades técnicas y funciones de cada uno de los individuos así como entablar confianza con los mismos.

La baja escolaridad también podría mediar la claridad de las emociones y el cómo éstas son influidas por los acontecimientos y entornos sociales, además del reconocimiento y expresión de sentimientos adecuados al entorno laboral. El conocimiento procede de las experiencias propiciadas por el contexto, y la forma de manifestar distintos tipos de emociones, dependiendo del repertorio previo y de la situación. Esto lleva a que se originen ganancias importantes en la comprensión, regulación emocional y respuesta empática (Henao \& García, 2009). 
En cuanto al nivel de estudios, hay investigaciones que han correlacionado el locus de control con el nivel de escolaridad, y ha en los que se ha encontrado que a menor nivel de preparación, el control es externo, y a mayor nivel de preparación, el control es interno (Encinas, Díaz, Noriega, \& Estrada, 2009). En este aspecto y debido a que los resultados nos reportan poca cantidad de sujetos con estudios avanzados, suponemos que el locus de control de estos sujetos de estudio es externo (aspecto que valdría la pena estudiar) y que, por lo tanto, carecen de habilidades de autoconciencia, lo que reitera que el instrumento de autoinforme TMMS-24 no es el más adecuado para este tipo de muestra.

Los niveles inferiores en adaptabilidad social, por su parte, denotan una falta de habilidades necesarias para la administración de personal, misma incompetencia que puede causar pérdida de motivación de los trabajadores, baja moral en los empleados, incumplimiento de deberes laborales en tiempo y forma, disminución de productividad y problemas disciplinares debido a inexistencia de supervisión, renuncias constantes $\mathrm{y}$, por ende, detrimento económico de la compañía. Este último debido al pago de salario industrial por salidas o bajas, remplazo constante de empleados y gastos que conllevan la capacitación (Deeb, 2015).

Esta falta de engranaje entre gerente y perfil es producto del incumplimiento de funciones de los procesos de selección de personal, ya que la contratación muchas veces está inmersa en la corrupción y el poco apego a los procedimientos de selección de personal por parte de Recursos Humanos, ya sea por permitir contrataciones a partir de relaciones de amistad con el interesado, por la influencia de los dueños o gerentes (Naranjo, 2012).

\section{Referencias}

Aburto, H., \& Bonales, J. (2011). Habilidades directivas: Determinantes en el clima organizacional. Investigación y Ciencia, 19(51), 41-49. Recuperado de http://www.redalyc. org/articulo.oa? $\mathrm{id}=67418397006$

Aguilar, Y., Valdez, J., González-López, N., \& González, S. (2013). Los roles de género de los hombres y las mujeres en el México contemporáneo. Enseñanza e Investigación en Psicología, 18(2), 207-224. Recuperado de http://www.redalyc.org/articulo.oa?id=2922 8336001

Aguilar, J., Ambrocio, H., \& Vargas, J. (2011). Reclutamiento y selección de personal: Procesos auditables y transparentes. Centro Regional de Investigación en Psicología, 5(1), 91-104. Recuperado de http://www.conductitlan.net/centro_regional_investigacion_psicologia/69_seleccion_personal_procesos_ auditables_transparentes.pdf

Alasa, A., \& García, R. (2010). Perfil de inteligencia emocional y carreras universitarias en estudiantes de la Universidad Simón Bolívar. Revista de Ciencias Sociales. 6(2), 226-238. Recuperado de: http://www.scielo.org.ve/ 
scielo.php?script $=$ sci_arttext\&pid $=\mathrm{S} 1315$ 95182010000200004\&lng=es\&nrm=iso

Bar-On, R. (2006). The Bar-On model of emotional-social intelligence (ESI). Psicothema, 18, 13-25. Recuperado de http://www.eiconsortium.org/pdf/baron_model_of_emotional_social_intelligence.pdf

Belinchón, I. (2006). La imagen de la empresa y de la marca en el mercado laboral. Anales de mecánica y electricidad, 82(05), 46-51. Recuperado de http://dialnet.unirioja.es/servlet/ articulo? codigo $=2136767$

Bernarás, E., Garaigordobil, M., \& De las Cuevas, C. (2011). Inteligencia emocional y rasgos de personalidad Influencia de la edad y el género durante la edad adulta y la vejez. Boletín de Psicología, 103, 75-88. Recuperado de http://www.uv.es/seoane/boletin/previos/ N103-5.pdf

Blasco, F., Fernández, S., \& Rodríguez, A. (2014). Employer branding: Estudio multinacional sobre la construcción de la marca del empleador. Universia Business Review, 44, 34-53. Recuperado de https://ubr.universia. net/index.php/ubr/article/view/974

Bosque, A., \& Aragón, L. (2008). Nivel de adaptación en adolescentes mexicanos. Interamerican Journal of Psychology, 42(2), 287-297. Recuperado de http:// pepsic.bvsalud.org/scielo.php? script $=$ sci arttext\&pid=S0034-96902008000200010\&ln $\mathrm{g}=\mathrm{pt} \& \mathrm{tlng}=\mathrm{es}$.

Calderón, G. (2003). Dirección de recursos humanos y competitividad. Innovar, 13(22), 157-
172. Recuperado dehttp://www.scielo.org.co/ scielo.php?script=sci_arttext\&pid=S0121$50512003000200013 \& \operatorname{lng}=\mathrm{en} \& \operatorname{tn} \mathrm{ln}=\mathrm{es}$

Camacho, J. (2008). Asociación entre variables cuantitativas: Análisis de correlación. Acta Médica Costarricense, 50(2), 94-96. Recuperado de http://www.scielo.sa.cr/ scielo.php?script $=$ sci_arttext\&pid $=$ S0001$60022008000200005 \& \operatorname{lng}=$ en\&tlng $=\mathrm{es}$

Cetina, T., Ortega, I., \& Aguilar, C. (2010). Habilidades Directivas desde la Percepción de los Subordinados: Un Enfoque Relacional para el Estudio del Liderazgo. Psicoperspectivas 9(1), 124-137. Recuperado de http://www.psicoperspectivas.cl/index. php/psicoperspectivas/article/viewFile/94/99 Chiavenato, I. (2000). Administración de recursos humanos. México: McGrawHill.

Crespo, N. (2000). La Metacognición: Las diferentes vertientes de una Teoría. Revista signos, 33(48), 97-115. Recuperado de http://www.scielo.cl/scielo.php?script $=$ sci arttext\&pid=S0718-09342000004800008\&ln $\mathrm{g}=\mathrm{es} \& \mathrm{tlng}=\mathrm{es} .10 .4067 / \mathrm{S} 0718-093420000048$ 00008.

Crissien, J. (2005). Gerencia del siglo XXI. Revista Escuela de Administración de Negocios, 54, 59-83. Recuperado de http://www. hacienda.go.cr/cifh/sidovih/spaw2/uploads/ images/file/Gerencia\%20del\%20Siglo\%20 XXI.pdf

Cuadra, A., \& Veloso, C. (2010). Grado de supervisión como variable moderadora entre liderazgo y satisfacción, motivación 
y clima organizacional. Ingeniare. Revista chilena de ingeniería, 18(1), 1525. Recuperado de http://www.scielo.cl/ scielo.php?script $=$ sci_arttext\&pid $=\mathrm{S} 0718$ $33052010000100003 \& \operatorname{lng}=\mathrm{es} \& \mathrm{t} \operatorname{lng}=\mathrm{es}$. 10.4067/S0718-33052010000100003.

Deeb, C. (2015). ¿Qué le ocurre a una organización con una mala administración? La Voz de Houston and the Houston Chronicle. [Traducido por Guilleron, L.]. Recuperado de http://pyme.lavoztx.com/qu-le-ocurreuna-organizacin-con-una-mala-administracin-13402.html

Enebral, J. (2001). Medida de la inteligencia emocional de las personas y de las organizaciones. Revista del Conocimiento, 12. Recuperado de http://www.geocities.ws/ccarbo/yacambu/egmrt/asignaturas/gdc/inteligenciaemocional/10.html

Encinas, D., Díaz, C., Noriega, J., \& Estrada, D. (2009). Locus de control y logro académico en dos tipos de ambiente de enseñanza para estudiantes universitarios. Pesquisas e Práticas Psicossociais, 3(2), 167-174. Recuperado de http://www.ufsj.edu.br/portal-repositorio/File/Encinas_etal.pdf

Extremera, N., Durán, A., \& Rey, L. (2005). La inteligencia emocional percibida y su influencia sobre la satisfacción vital, la felicidad subjetiva y el engagement en trabajadores de centros para personas con discapacidad intelectual. Ansiedad y Estrés, 11(1), 63-73.

Extremera, N., \& Fernández-Berrocal, P. (2004). El papel de la inteligencia emocional en el alumnado: Evidencias empíricas. Revista Electrónica de Investigación Educativa, 6(2). Recuperado de http://redie.uabc.mx/ vol6no2/contenido-extremera.html

Fernández, P., \& Ruiz, D. (2008). La Inteligencia emocional en la Educación. Revista Electrónica de Investigación Psicoeducativa, 6(2), 421-436. Recuperado de http://www. investigacion-psicopedagogica.org/revista/ new/ContadorArticulo.php?256

Fernandez, P., \& Extremera, N. (2009). La Inteligencia Emocional y el estudio de la felicidad. Revista Interuniversitaria de Formación del Profesorado, 66(23,3), 85-108.

Fernandez, P., \& Extremera, N. (2006). La investigación de la inteligencia emocional en España. Ansiedad y estrés, 12(2-3), 139-153. Recuperado de http://emotional.intelligence. uma.es/documentos/pdf53Inteligencia_ Emocional_en_Espana.pdf

Fernández, P., Extremera, N., \& Ramos, N. (2004). Validity and reliability of the Spanish modified version of the Trait Meta-Mood Scale. Psychological Reports, 94, 751-755.

Ferragut, M., \& Fierro, A. (2012). Inteligencia emocional, bienestar personal y rendimiento académico en preadolescentes. Revista Latinoamericana de Psicología, 44(3), 95104. Recuperado de http://www.redalyc.org/ pdf/805/80525022008.pdf

Fierro, A. (2006). Bienestar personal, adaptación social y factores de personalidad: Estudios con las Escalas Eudemon. Clinica y salud 17(3). Recuperado de http://scielo. 
isciii.es/scielo.php?script=sci_arttext\&pid $=\mathrm{S} 1130-52742006000300006$

García, M., Gómez, P., \& Londoño, O. (2009). Relación entre motivación y resistencia al cambio en personas que trabajan en una empresa del sector público, en Bogotá (Colombia). Diversitas: Perspectivas en Psicología, 5(1), 141-159. Recuperado de http://www.scielo.org.co/ scielo.php?script $=$ sci_arttext\&pid $=\mathrm{S} 1794$ 99982009000100012\&lng=en\&tlng=es

Goleman, D. (1995). Inteligencia emocional. Barcelona: Kairós.

Goleman, D. (1999). La Inteligencia Emocional en la Empresa. Colombia: Ediciones B Argentina, S. A. para el sello Javier Vergara Editor.

Henao, G., \& García, M. (2009). Interacción familiar y desarrollo emocional en niños y niñas. Revista Latinoamericana de Ciencias Sociales, Niñez y Juventud, 7(2), 785-802. Recuperado de http://www.scielo.org.co/ scielo.php?script=sci_arttext\&pid=S1692$715 X 2009000200009 \& \operatorname{lng}=$ en\&tlng=es.

Hernández, R., Silvestri, K., Añez, S., \& Gamboa, L. (2008). Realidad de la formación gerencial en las pequeñas y medianas empresas venezolanas. Revista Venezolana de Gerencia 13(41), 107-125. Recuperado de http:// www.scielo.org.ve/pdf/rvg/v13n41/art07.pdf Lewis, S., Cuesta, M., Ghisays, Y., \& Romero, L. (2004). La adaptación social y escolar en niños con y sin trastorno por déficit de atención con hiperactividad (TDAH) de la ciudad de
Barranquilla (Colombia). Psicología desde el Caribe, 14, 125-149. Recuperado de http:// www.redalyc.org/articulo.oa?id=21301406 López R., \& Martínez, A. (2014). Inteligencia emocional y la ventana del líder en los directivos turísticos. Ing. Ind. 35(2), 229239. Recuperado de http://scielo.sld.cu/ scielo.php?script $=$ sci_arttext\&pid $=\mathrm{S} 1815$ 59362014000200010\&lng=es\&nrm=iso

Mirza, C., Núñez, M., \& Rodríguez, C. (2011). Factores grupales que inciden en la productividad. Universidad, Ciencia y Tecnología, 15(61), 223-231. Recuperado de http://www.scielo.org.ve/ scielo.php?script $=$ sci_arttext\&pid $=$ S131648212011000400006\&lng=es\&tlng=es

Naranjo, R. (2012). El proceso de selección y contratación del personal en las medianas empresas de la ciudad de Barranquilla (Colombia). Pensamiento y gestión 32, 83-114. Recuperado de http://rcientificas.uninorte. edu.co/index.php/pensamiento/article/viewFile/3978/2826

Oriolo, E., \& Cooper, R. (1998). La inteligencia emocional aplicada al liderazgo y a la organización. Bogotá, Colombia: Grupo Editorial Norma.

Ortiz, R., \& Beltrán, B. (2011). Inteligencia emocional percibida y desgaste laboral en médicos internos de pregrado. Educ. Méd. 14(1). Recuperado de http://scielo.isciii.es/ scielo.php?script $=$ sci_arttext\&pid $=$ S157518132011000100010\&lng=es. 
Osses, S., \& Jaramillo, S. (2008). Metacognición: Un camino para aprender a aprender. Estudios Pedagógicos 34(1), 187-197. Recuperado de http://www.scielo.cl/pdf/estped/ v34n1/art11.pdf

Padrón, G., \& Sánchez, M. (2010). Efecto de la motivación al logro y la inteligencia emocional en el Crecimiento Psicológico. Revista Venezolana de Gerencia, 15(49), 141-158. Recuperado de http:/www.scielo.org.ve/ scielo.php?script $=$ sci_arttext\&pid $=\mathrm{S} 1315$ 99842010000100009\&lng=es\&tlng=es

Pena, M., \& Repetto, E. (2008). Estado de la investigación en España sobre Inteligencia Emocional en el ámbito educativo. Revista Electrónica de Investigación Psicoeducativa, 6(2). 400-420.

Perandones, T., \& Castejón, J. (2007). Estudio correlacional entre personalidad, inteligencia emocional y autoeficacia en profesorado de educación secundaria y bachillerato. Libro de actas V Congreso Internacional Educación y Sociedad. La Educación: Retos del S. XXI, 1-8. Granada, Universidad de Alicante.

Real Academia Española. (2015). Recuperado de http://lema.rae.es/drae/

Robbins, S. (1999). Comportamiento organizacional. México: Prentice Hall.

Rodríguez de Torrealba, A. (2008). Hacia una visión prospectiva de la gerencia y supervisión educativa. Investigación y Postgrado, 23(2), 447-461. Recuperado de http:// www.scielo.org.ve/scielo.php? script $={ }$ sci $_{-}$ arttext\&pid $=\mathrm{S} 1316-00872008000200017 \& \ln$ $\mathrm{g}=\mathrm{es} \& \mathrm{t} \operatorname{lng}=\mathrm{es}$.

Salovey, P., \& Mayer, J. D. (1997). What is Emotional intelligence? In P. Salovey \& D. J. Sluyter (Eds.), Emotional Development and Emotional Intellingence. New York: Basic Books.

Sanjuán, A., \& Ferrer, M. (2008). Perfil emocional de los estudiantes en prácticas clínicas: Acción tutorial en enfermería para apoyo, formación, desarrollo y control de las emociones. Investigación y Educación en Enfermería, 26(2), 226-235. Recuperado de http:/www.scielo.org.co/ scielo.php? script $=$ sci_arttext\&pid $=\mathrm{S} 0120$ $53072008000200005 \& \operatorname{lng}=$ en\&tlng=es

Subuyuj, W. (2013). Adaptabilidad social de un grupo de auditores que laboran en distintas empresas de la ciudad de Guatemala. Guatemala de la Asunción: Universidad Rafael Landívar Facultad de Humanidades.

Trujillo, M., \& Rivas, L. (2005). Orígenes, evolución y modelos de inteligencia emocional. Innovar, 15(25), 9-24. Recuperado de http://www.scielo.org.co/ scielo.php? script $=$ sci_arttext\&pid $=$ S0121 $50512005000100001 \& \operatorname{lng}=$ en\&tlng=es

Veloso, C., Cuadra A., Antezana, I., Avendaño, R., \& Fuentes, L. (2013). Relación entre inteligencia emocional, satisfacción vital, felicidad subjetiva y resiliencia en funcionarios de educación especial. Estudios Pedagógicos, 39(2), 355366. Recuperado de http://www.scielo.cl/ 
scielo.php?script $=$ sci_arttext\&pid $=\mathrm{S} 0718$ 07052013000200022\&lng=es\&tlng=es. 10.4067/S0718-07052013000200022.

Vera, A., Cancino, L., Ravanal, I., Carrasco, C., Contreras, G., \& Arteaga, O. (2007). Síndrome de burnout e inteligencia emocional: Un enfoque psicosocial en una agencia estatal chilena. Ciencia \& Trabajo, 24, 51-54.

Zarate, R., \& Matviuk, S. (2012). Inteligencia emocional y prácticas de liderazgo en las organizaciones colombianas. Cuadernos de administración, 28(47), 91-104. Recuperado de http://www.scielo.org.co/pdf/cuadm/ v28n47/v28n47a08.pdf

Recibido el 23 de noviembre de 2015

Revisado el 19 de febrero de 2016

Aceptado el 27 de abril de 2016 